\title{
LESÃO DERMONECRÓTICA EM UM GATO ATRIBUÍDA A ENVENENAMENTO POR LOXOSCELES - RELATO DE CASO
}

\author{
DERMONECROTIC LESION IN A CAT ATTRIBUTED TO LOXOSCELES ENVENOMATION - \\ CASE REPORT
}

\author{
K. O. DUARTE ${ }^{1}$, L. BALLARDIN ${ }^{2}$, N. T. VIEIRA ${ }^{3}$, A. L. C. TERRA ${ }^{4}$
}

\begin{abstract}
RESUMO
Os acidentes causados por aranhas do gênero Loxosceles têm importância na clínica de pequenos animais, mesmo não havendo dados epidemiológicos desses ataques em animais de estimação, dada a gravidade da lesão e possíveis complicações sistêmicas resultantes, tornando indispensável maior conhecimento sobre a espécie e as consequências do envenenamento. $\mathrm{O}$ presente trabalho relata a presença de uma lesão dermonecrótica em um felino atribuída a aranhamarrom (Loxosceles sp). O atendimento ocorreu na clínica Empório de Bicho em Caxias do Sul, no estado do Rio Grande do Sul. O animal apresentava uma lesão necrótica em região perineal, com fibrina e secreção purulenta. $\mathrm{O}$ tratamento consistiu no uso clorexidina para limpeza, hidrogel, açúcar, Fitofix ${ }^{\circledR}$ e Dersani ${ }^{\circledR}$ como promotores de cicatrização. Após um mês de tratamento, o animal apresentava uma melhora satisfatória.
\end{abstract}

PALAVRAS-CHAVE: Aranha-marrom. Dermonecrose. Gato. Loxosceles.

\section{SUMMARY}

Accidents caused by spiders of the genus Loxosceles are important in the small animal clinic, even though there are no epidemiological data on these attacks in pets, given the severity of the lesion and possible systemic complications, it becomes necessary to know more about the species and the consequences of the poisoning. The present work reports the presence of a dermonecrotic lesion in a cat attributed to brown spider (Loxosceles sp). The patient had a necrotic lesion in the perineal region with fibrin and purulent secretion. The treatment consisted of using chlorhexidine for cleaning, hydrogel, sugar, Fitofix ${ }^{\circledR}$ and Dersani ${ }^{\circledR}$ as healing promoters. After one month of treatment, the animal showed a satisfactory improvement.

KEY-WORDS: Brown spider. Cat. Dermonecrosis. Loxosceles.

\footnotetext{
${ }^{1}$ Universidade de Caxias do Sul. Email.: kimberli.duarte@ gmail.com

${ }^{2}$ Universidade de Caxias do Sul. Email.: contato@emporiodebicho.com.br

${ }^{3}$ Universidade Federal de Rondônia. Email.: nayche@unir.br

${ }^{4}$ Universidade Federal de Rondônia. Email.: prof.angeloterra@gmail.com
} 


\section{INTRODUÇ̃̃O}

A aranha-marrom é um animal de hábitos noturnos que evita a presença humana. No ambiente domiciliar, ela se abriga em camas, porões, sob pilhas de roupas (HOVDA et al., 2016). Essas aranhas não são agressivas e picam apenas quando ameaçadas (PETERSON e MCNALLY, 2013).

A toxina esfingomielinase D atua sobre a esfingomielina das membranas endoteliais, hemácias e plaquetas, causando a ruptura destas, tem ação também na ativação da cascata de complemento, lise celular, apoptose e uma resposta imune que leva à dermonecrose. Atua como fator quimiotático para neutrófilos, acarretando o recrutamento dessas células inflamatórias para o local da lesão. O influxo de neutrófilos e a lise das células do endotélio capilar promovem a agregação plaquetária, resultando em coagulação intravascular rápida nos capilares ao redor da lesão produzindo oclusão de pequenos capilares e necrose dérmica (SAKATE, 2008; HOVDA et al., 2016).

O loxoscelismo se apresenta em duas formas sendo a cutânea a mais observada na maioria dos casos, caracterizando-se pelo aparecimento de lesão inflamatória no ponto da picada, com edema leve e eritema evoluindo para necrose e ulceração. Uma escara se forma sobre a área, cobrindo uma ferida ulcerante profunda com cicatrização lenta e possibilidade de infecção secundária (HOVDA et al., 2016). A viscerocutânea, na qual verifica-se que os animais acometidos apresentam anemia, icterícia, hemoglobinúria, hematúria e trombocitopenia. A insuficiência renal aguda é resultado, entre outros fatores, da ação nefrotóxica e hemolítica do veneno (SAKATE, 2008). Leucocitose, anemia hemolítica, trombocitopenia, tempos de coagulação prolongados, elevações nas enzimas hepáticas podem estar presentes (HOVDA et al., 2016).

Devido à existência de diversos relatos de casos de loxoscelismo em caninos, a sintomatologia é bem conhecida nesses pacientes, contudo, em gatos não existe literatura publicada relatando a manifestação clínica causada pelo veneno da aranha, tampouco há relatos sobre qual tratamento seria mais efetivo nos pacientes felinos.

O tratamento deverá restringir-se ao uso de anti-inflamatório não-esteroidal, excetuando o ácido acetilsalicílico, pois este poderá agravar as alterações sanguíneas e é tóxico para esta espécie (SAKATE, 2008). Os corticosteroides sistêmicos têm um efeito protetor na membrana dos eritrócitos, inibindo a hemólise (PETERSON e MCNALLY, 2013). No local da picada, recomenda-se realizar compressas frias e antissépticas. Os antibióticos devem ser empregados por via sistêmica, se houver infecções secundárias (SAKATE, 2008). Recomenda-se também limpeza da ferida com água e sabão, podendo ser necessário algum debridamento (PETERSON e MCNALLY, 2013). A recuperação total pode levar de semanas a meses, mas o prognóstico é bom se os sinais sistêmicos não forem observados (HOVDA et al., 2016).

\section{RELATO DE CASO}

Um felino de 2 anos, com 4,3 kg, sem raça definida foi atendido na Clínica Veterinária Empório de Bicho, no dia 18 de junho de 2017 (Dia 1). Neste primeiro atendimento o animal apresentava apatia, anorexia, vômitos, febre, desconforto abdominal, tempo de perfusão capilar aumentado e desidratação de $6 \%$. O hemograma revelou eritrocitose, trombocitopenia, leucopenia por neutrofilia e linfopenia com presença de agregado plaquetário. Nos exames bioquímicos nenhuma alteração foi encontrada em ALT, albumina, creatinina, FA, GGT e ureia. Na ultrassonografia foi observada discreta indefinição corticomedular condizente com nefropatia incipiente/leve, e fígado com parênquima hipoecogênico homogêneo compatível com hepatopatia e/ou toxemia.

O hemograma foi repetido dois dias depois e ficou constatado um agravamento da trombocitopenia, e a linfopenia com agregado plaquetário se mostrava persistente.

No terceiro retorno à clínica, o tutor retornou com o felino apresentando uma lesão em região perineal. No dia seguinte foi repetido o hemograma que persistia com trombocitopenia, a linfopenia já não estava presente, mas uma leucocitose por neutrofilia com agregados plaquetários pode ser observada. No mesmo dia novo exame ultrassonográfico foi realizado constatando-se um aumento de volume em região inguinal caudal direita decorrente de aumento de espessura de tecido subcutâneo, caracterizando edema neste caso.

No retorno quarto retorno, a lesão já se mostrava necrótica com secreção purulenta e com presença de fibrina (Figura 1). Após a visualização de lesão e relato do proprietário informando que uma outra aranha com as características da Loxosceles havia aparecido em sua residência estabeleceu-se diagnóstico presuntivo de loxocelismo. $\mathrm{O}$ paciente também apresentava vômito e dor aguda. Optou-se por tratamento de ferida aberta com clorexidina solução aquosa e hidrogel (água, carboximetilcelulose e propilenoglicol). $\mathrm{O}$ antibiótico utilizado neste caso foi a amoxicilina com clavulanato.

No sexto retorno a lesão já se apresentava sem fibrina e com granuloma de cicatrização. Indicou-se a continuidade com clorexidina, hidrogel, açúcar e Fitofix ${ }^{\circledR}$ (Tintura de Calendula officinalis L., tintura de Stryphnodendron barbatiman Mart., tintura de Symphyntum officinalis L., tintura de Aloe vera, tintura de Matricaria chamomilla L., tintura de Echinacea angustifolia DC., tintura de própolis). Três dias após o tecido cicatricial estava bem ativo e foi suspendido o hidrogel devido à presença do tecido de granulação.

Um mês após a lesão continuava com evolução positiva na cicatrização, sendo mantida somente a loção oleosa Dersani ${ }^{\circledR}$ (Triglicerídeos de ácidos cáprico e caprílico, óleo de girassol clarificado, lecitina, palmitato de retinol, acetato de tocoferol e alfa-tocoferol) esporadicamente (Figura 2) acompanhada da retirada de depósitos fibrinonecróticos aderidos ao leito. 


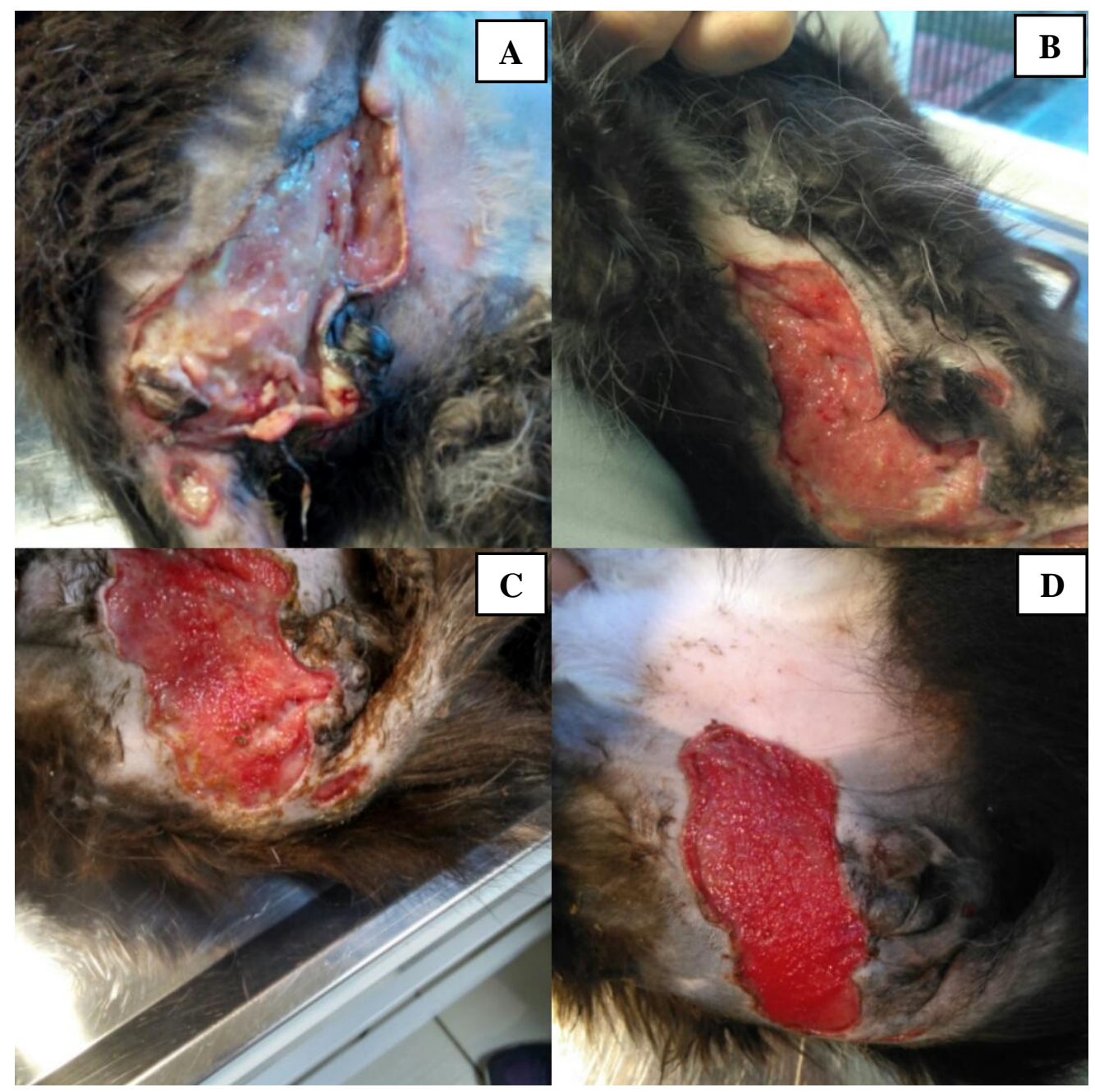

Figura 1 - Evolução da lesão. Fotos retiradas durante os retornos do tutor à clínica. A: Dia 5, B: Dia 6, C: Dia 7, D: Dia 8.

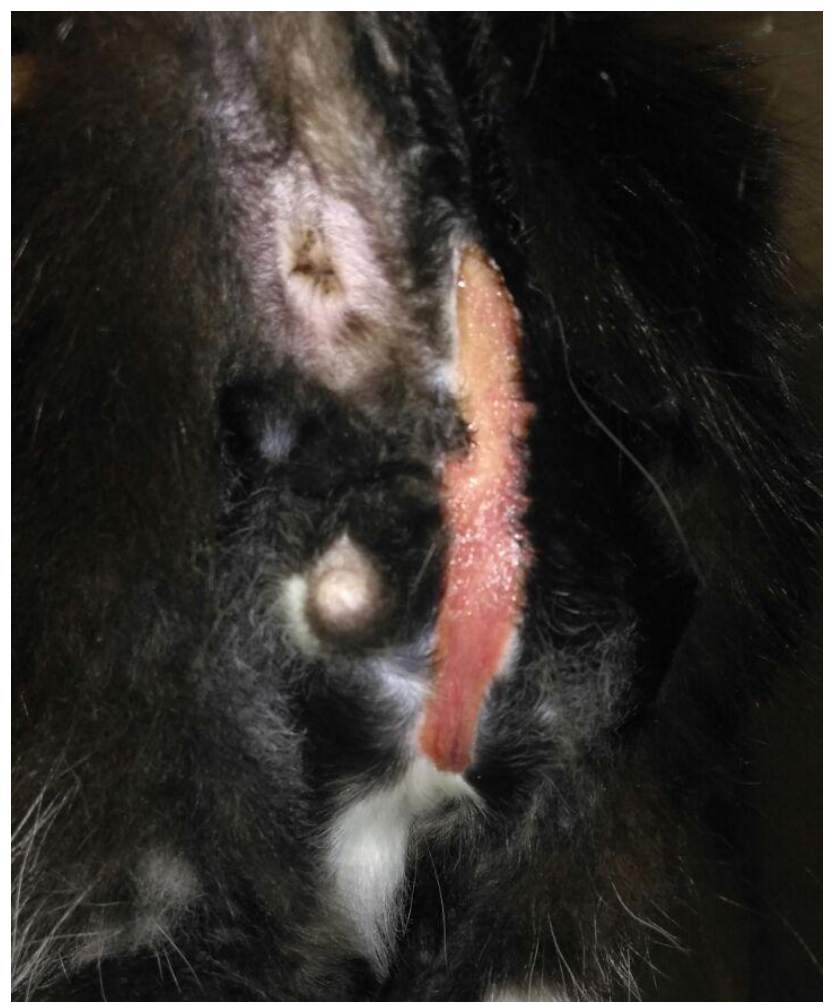

Figura 2 - Fotografia do último retorno do tutor com o paciente à clínica. Aproximadamente um mês após a consulta inicial (Dia 9). 


\section{DISCUSSÃO}

Segundo Chagas et al., (2010) o período de maior casuística em humanos foi de outubro a abril, corroborando com o que Ribeiro et al., (1993) haviam publicado. Entretanto, o agravo ao animal ocorreu em junho (inverno). Sugere-se que essa divergência possa ser explicada pela reforma na residência do tutor, o que poderia ter induzido o ataque.

Corroborando a literatura, o animal apresentou trombocitopenia. A discrepância nesse caso, seria leucopenia com neutrofilia e linfopenia, já que Hovda et al., (2016) e Peterson e Mcnally (2013) relatam a ocorrência de uma leucocitose, podendo a ocorrência de tal achado ser em detrimento do recrutamento do reservatório circulante mediante agressão tecidual focal.

Chatzaki et al., (2012) relatam que o veneno causou degeneração e necrose após oito horas em um estudo in vivo realizado com coelhos. No paciente felino, a dermonecrose levou aproximadamente seis dias para aparecer.

Os sinais clínicos apresentados pelo paciente (apatia, anorexia, vômitos, febre, dor local) são os mesmos relatados por Sakate (2008). O tempo de tratamento longo é relatado por diversos autores tais como Gfeller e Messonnier (2003) e Roder (2003), entretanto se sinais sistêmicos estiverem ausentes, o prognóstico é favorável (HOVDA et al., 2016).

Como o tratamento específico com soro antiloxoscélico não está disponível para uso animal, sugere-se como terapêutica de escolha fornecer cuidado sintomático e de suporte, com a limpeza da lesão e utilização de pomadas que ajudem na cicatrização (SAKATE, 2008; HOVDA et al., 2016). Como a lesão do paciente foi muito extensa e profunda, tornou-se necessário a utilização de uma combinação de pomadas e substâncias para melhora rápida e satisfatória.

O diagnóstico definitivo seria a visualização da Loxosceles, porém essas aranhas atacam e fogem imediatamente, o que dificulta a identificação precisa (HOVDA et al., 2016). Consequentemente a maioria dos casos é feito com o diagnóstico presuntivo, excluindo outras possíveis causas (MÁLAQUE, 2002).

Como foi relatado pelo tutor o aparecimento de outra aranha, foi sugerido a este um conjunto de ações para eliminar os aracnídeos da residência. Já foi relatado com sucesso a utilização de aspiradores de pó (RAMIRES et al., 2007), preservação de predadores naturais como a lagartixa (RAMIRES e FRAGUAS, 2004) e até armadilhas para aranhas (PARKS et al., 2013). O tutor do caso relatado decidiu sair da residência por alguns dias e fazer uma dedetização completa na moradia.

Existem diversos relatos de loxoscelismo na medicina veterinária, como relatam Collacico et al., (2008), Machado et al., (2009), entre outros autores. Entretanto, todos os relatos descrevem os acidentes em cães. $\mathrm{O}$ acometimento em felinos é raro já que não existem relatos de ataques na espécie. $\mathrm{O}$ estudo desses ataques em gatos é importante para o entendimento das diferenças entre as espécies e as possíveis divergências entre tratamentos, visando uma melhor recuperação e maior sobrevida nos animais acometidos com a doença na forma sistêmica.

\section{CONSIDERAÇÕES FINAIS}

O caso relatado demonstra a importância de uma abordagem terapêutica rápida e adequada, pois embora os acidentes por Loxosceles raramente levem a óbito, é importante seguir a conduta clínica correta para evitar lesões muito extensas que aumentam o tempo até a total cicatrização. Em conjunto com o tratamento, é essencial a prevenção de novos acidentes eliminando os aracnídeos.

Cabe salientar ainda a necessidade de realizar estudos epidemiológicos e toxinológicos em medicina preventiva e saúde pública, para que se possa entender a prevalência desses animais e, dessa forma, realizar ações preventivas mais efetivas.

A ausência de notificações de loxocelismo na espécie felina justificou o relato deste caso fornecendo informações clínicas e científicas que serão úteis na elaboração de tratamentos em outros casos que vierem a ser registrados.

\section{AGRADECIMENTOS}

Agradeço à equipe da clínica veterinária Empório de Bicho pela colaboração na disponibilização dos dados da anamnese, procedimentos clínicos e evolução do quadro do paciente, fundamentais para realização deste trabalho. Agradeço também ao sr. Otávio Martins, proprietário do felino "Kimi", pela dedicação e comprometimento demonstrados durante o tratamento.

\section{REFERÊNCIAS}

CHAGAS, F. B.; D'AGOSTINI, F. M.; BETRAME, V. Aspectos epidemiológicos dos acidentes por aranhas no Estado do Rio Grande do Sul, Brasil. Evidência, v. 10 n. $1-2$, p. 121-130, janeiro/dezembro 2010. DOI: 10.13140/RG.2.1.2249.6400

CHATZAKI, M.; HORTA, C.C.; ALMEIDA, M.O.; PEREIRA, N.B.; MENDES, T.M.; DIAS-LOPES, C.; GUIMARÃES, G.; MORO, L.; CHÁVEZOLÓRTEGUI, C.; HORTA, M.C.R.; KALAPOTHAKIS, E. Cutaneous loxoscelism caused by Loxosceles similis venom and neutralization capacity of its specific antivenom. Toxicon, [s.1.], v. 60, n. 1, p.21-30, jul. 2012.

COLLACICO, K.; CHANQUETTI, A.M.S.; FERRARI, R. Acidente por loxosceles em cão - relato de caso. Ensaios e Ciência: Ciências Biológicas, Agrárias e da Saúde. 2008, XII.

GFELLER, R.W.; MESSONNIER, S.P. Handbook of Small Animal Toxicology and Poisonings. 2. ed. St. Louis: Mosby, 2003. 512 p. 
HOVDA, L. R.; BRUTLAG, A.G.; POPPENGA, R.H.; PETERSON, K.L. Blackwell's Five-Minute Veterinary Consult Clinical Companion: Small Animal Toxicology. Ames, Iowa: John Wiley e Sons, 2016.

MACHADO, LHA; Antunes, M.I.P.P.; Mazini, A.M.; Sakate, M.; Torres-Neto R.; Fabris V.E.; Vailati M.C.F.; Lourenço M.L.G. Necrotic skin lesion in a dog attributed to Loxosceles (brown spider) bite: a case report. J. Venom. Anim. Toxins incl. Trop. Dis [online]. 2009, vol.15, n.3 [cited 2018-08-25], pp.572-581.

MÁLAQUE， C.M.S.; CASTRO-VALENCIA， J.E.; CARDOSO, J.L.C.; FRANÇA, F.O.S.; BARBARO, K.C.; FAN, H.W. Clinical and epidemiological features of definitive and presumed loxoscelism in São Paulo, Brazil. Rev. Inst. Med. trop. S. Paulo, São Paulo, v. 44, n. 3, p. 139-143, 2002.

PARKS, J.; PAIGE, R.L.; STOECKER, W.V. Trap Design for the Brown Recluse Spider, Loxosceles reclusa. Journal Of Insect Science, v. 13, n. 57, p.1-6, jun. 2013. Oxford University Press (OUP). http://dx.doi.org/10.1673/031.013.5701.

PETERSON, M. E.; MCNALLY, J. Spider Envenomation: Brown Recluse. In: PETERSON, M. E.; TALCOTT, P. A. Small Animal Toxicology. 3. ed. St. Louis: Saunders, 2013. Cap. 80. p. 823-826.
RODER, J. D. Spiders. In: PLUMLEE, K. Clinical Veterinary Toxicology. St. Louis: Mosby, 2003. Cap. 3. p. 112-113.

RAMIRES, E. N.; RETZLAFF, A.V.L.; DECONTO, L.R.; FONTANA, J.D.; MARQUES, F.A.; MARQUES-DA-SILVA, E. Evaluation of the efficacy of vacuum cleaners for the integrated control of brown spider Loxosceles intermedia. J. Venom. Anim. Toxins incl. Trop. Dis., 2007, 13, 3, p. 619. http://dx.doi.org/10.1590/S1678-91992007000300005

RAMIRES, E.N.; FRAGUAS, G.M. Tropical House Gecko (Hemidactylus mabouia) predation on brown spiders (Loxosceles intermedia). J. Venom. Anim. Toxins incl. Trop. Dis [online]. 2004, vol.10, n.2, pp.185-190. ISSN 1678-9199. http://dx.doi.org/10.1590/S1678-91992004000200008.

RIBEIRO, L.A.; EICKSTEDT, V.R.D.; RÚBIO, G.B.G.; KONOLSAISEN, J.F.; HANDAR, Z.; ENTRES, M.; CAMPOS, V.A.F.P.; JORGE, M.T. Epidemiologia do acidente por aranhas do gênero Loxosceles Heinecken e Lowe no Estado do Paraná (Brasil). Mem. Inst. Butantan, v.55, n.1, p. 19-26, 1993.

SAKATE, Michiko. Zootoxinas. In: SPINOSA, H.S.; GÓRNIAK, S.L.; PALERMO-NETO, J. Toxicologia aplicada à medicina veterinária. Barueri: Manole, 2008. Cap. 8. p. 240-242. 